\title{
Electricity load forecasting: a systematic review
}

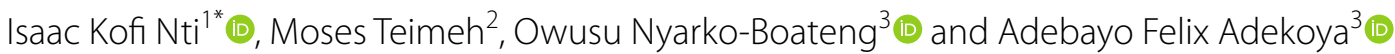

\section{${ }^{*}$ Correspondence:}

ntious1@gmail.com

${ }^{1}$ Department of Computer

Science, Sunyani Technical

University, Box 206, Sunyani,

Ghana

Full list of author information

is available at the end of the

article

\begin{abstract}
The economic growth of every nation is highly related to its electricity infrastructure, network, and availability since electricity has become the central part of everyday life in this modern world. Hence, the global demand for electricity for residential and commercial purposes has seen an incredible increase. On the other side, electricity prices keep fluctuating over the past years and not mentioning the inadequacy in electricity generation to meet global demand. As a solution to this, numerous studies aimed at estimating future electrical energy demand for residential and commercial purposes to enable electricity generators, distributors, and suppliers to plan effectively ahead and promote energy conservation among the users. Notwithstanding, load forecasting is one of the major problems facing the power industry since the inception of electric power. The current study tried to undertake a systematic and critical review of about seventy-seven (77) relevant previous works reported in academic journals over nine years (2010-2020) in electricity demand forecasting. Specifically, attention was given to the following themes: (i) The forecasting algorithms used and their fitting ability in this field, (ii) the theories and factors affecting electricity consumption and the origin of research work, (iii) the relevant accuracy and error metrics applied in electricity load forecasting, and (iv) the forecasting period. The results revealed that $90 \%$ out of the top nine models used in electricity forecasting was artificial intelligence based, with artificial neural network (ANN) representing 28\%. In this scope, ANN models were primarily used for short-term electricity forecasting where electrical energy consumption patterns are complicated. Concerning the accuracy metrics used, it was observed that root-mean-square error (RMSE) (38\%) was the most used error metric among electricity forecasters, followed by mean absolute percentage error MAPE (35\%). The study further revealed that $50 \%$ of electricity demand forecasting was based on weather and economic parameters, $8.33 \%$ on household lifestyle, $38.33 \%$ on historical energy consumption, and 3.33\% on stock indices. Finally, we recap the challenges and opportunities for further research in electricity load forecasting locally and globally.
\end{abstract}

Keywords: Electricity load forecasting, Machine learning, Electrical energy demand, Artificial intelligence

\section{Background}

Electricity is the pivot in upholding highly technologically advanced industrialisation in every economy [1-3]. Almost every activity done in this modern era hinges on electricity. The demand and usage of electric energy increase globally as the years past

\section{SpringerOpen}

(c) The Author(s) 2020. This article is licensed under a Creative Commons Attribution 4.0 International License, which permits use, sharing, adaptation, distribution and reproduction in any medium or format, as long as you give appropriate credit to the original author(s) and the source, provide a link to the Creative Commons licence, and indicate if changes were made. The images or other third party material in this article are included in the article's Creative Commons licence, unless indicated otherwise in a credit line to the material. If material is not included in the article's Creative Commons licence and your intended use is not permitted by statutory regulation or exceeds the permitted use, you will need to obtain permission directly from the copyright holder. To view a copy of this licence, visit http://creativeco mmons.org/licenses/by/4.0/. 
[4]; however, the process of generating, transmitting, and distributing electrical energy remains complicated and costly. Hence, effective grid management is an essential role in reducing the cost of energy production and increased in generating the capacity to meet the growing demand in electric energy [5].

Accordingly, effective grid management involves proper load demand planning, adequate maintenance schedule for generating, transmission and distribution lines, and efficient load distribution through the supply lines. Therefore, an accurate load forecasting will go a long way to maximise the efficiency of the planning process in the power generation industries [5, 6]. As a means to improve the accuracy of Electrical Energy Demand (EED) forecasting, several computational and statistical techniques have been applied to enhance forecast models [7].

EED forecasting techniques can be clustered into three (3), namely correlation, extrapolation, and a combination of both. The Extrapolation techniques (Trend analysis) involve fitting trend curves to primary historical data of electrical energy demand in a way to mirror the growth trend itself $[7,8]$. Here, the future value of electricity demand is obtained from estimating the trend curve function at the preferred future point. Despite its simplicity, its results are very realistic in some instances [8].

On the other hand, correlation techniques (End-use and Economic models) involve relating the system load to several economic and demographic factors $[7,8]$. Thus, the techniques ensure that the analysts capture the association existing between load increase patterns and other measurable factors. However, the disadvantage lies in the forecasting of economic and demographic factors, which is more complicated than the load forecast itself $[7,8]$. Usually, economic and demographic factors such as population, building permits, heating, employment, ventilation, air conditioning system information, weather data, building structure, and business are used in correlation techniques [7-9]. Nevertheless, some researchers group EED forecasting models into two, viz. data-driven (artificial intelligence) methods (same as the extrapolation techniques) and engineering methods (same as correlation the techniques) [9]. All the same, no single method is accepted scientifically superior in all situations.

Also, proper planning and useful applications of electric load forecasting require particular "forecasting intervals," also referred to as "lead time". Based on the lead time, load forecasting can be grouped into four (4), namely: very short-term load forecasting (VSTLF), short-term load forecasting (STLF), medium-term load forecasting (MTLF) and long-term load forecasting (LTLF) $[6,7,10]$. The VSTLF is applicable in real-time control, and its predicting period is within minutes to $1 \mathrm{~h}$ ahead. The STLF is for making forecasting within $1 \mathrm{~h}$ to 7 days or month ahead [11]. It is usually used for the day-to-day operations of the utility industry, such as scheduling the generation and transmission of electric energy. The MTLF is used for forecasting of fuel purchase, maintenance, utility assessments. Its forecasting period ranges from 1 week to 1 year. While the LTLF is for making forecasting beyond a year to 20 years ahead, it is suitable for forecasting the construction of new generations, strategic planning, and changes in the electric energy supply and delivery system [10].

Notwithstanding the above-mentioned techniques and approaches available, EED forecasting is seen to be complicated and cannot easily be solved with simple mathematical formulas [2]. Also, Hong and Fan [12] pointed out that electric load forecasting 
has been a primary problem for the electric power industries, since the inception of the electric power. Regardless of the difficulty in electric load forecasting, the optimal and proficient economic set-up of electric power systems has continually occupied a vital position in the electric power industries [13]. This exercise permits the utility industries to examine the dynamic growth in load demand patterns to facilitate continuity planning for a better and accurate power system expansion. Consequently, inaccurate prediction leads to power shortage, which can lead to "dumsor" and unneeded development in the power system leading to unwanted expenditure [7, 14]. Besides, a robust EED forecasting is essential in developing countries having a low rate of electrification to facilitate a way for supporting the active development of the power systems [15].

Based on the sensitive nature of electricity demand forecasting in the power industries, there is a need for researchers and professionals to identify the challenges and opportunities in this area. Besides, as argued by Moher et al. [16], systematic reviews are the established reference for generating evidence in any research field for further studies. Our partial search of literature resulted in the following [10, 12, 17-21] papers that focused on comprehensive systematic review concerning the methods, models, and several methodologies used in electric load forecasting. Hammad et al. [10] compared forty-five (45) academic papers on electric load forecasting based on inputs, outputs, time frame, the scale of the project, and value. They revealed that despite the simplicity of regression models, they are mostly useful for long-term load forecasting compared with AI-based models such as ANN, Fuzzy logic, and SVM, which are appropriate for short-term forecasting.

Similarly, Hong and Fan [12] carried out a tutorial review of probabilistic EED forecasting. The paper focused on EED forecasting methodologies, special techniques, common misunderstandings and evaluation methods. Wang et al. [19] presented a comprehensive review of factors that affects EED forecasting, such as forecast model, evaluation metric, and input parameters. The paper reported that the commonly used evaluation metrics were the mean absolute error, MAPE, and RMSE. Likewise, Kuster et al. [22] presented a systematic review of 113 studies in electricity forecasting. The paper examined the timeframe, inputs, outputs, data sample size, scale, error type as criteria for comparing models aimed at identifying which model best suited for a case or scenario.

Also, Zhou et al. [17] presented a review of electric load classification in the smartgrid environment. The paper focused on the commonly used clustering techniques and well-known evaluation methods for EED forecasting. Another study in [21] presented a review of short-term EED forecasting based on artificial intelligence. Mele [20] presented an overview of the primary machine learning techniques used for furcating short-term EED. Gonzalez-Briones et al. [18] examined the critical machine learning models for EED forecasting using a 1-year dataset of a shoe store. Panda et al. [23] presented a comprehensive review of LTLF studies examining the various techniques and approaches adopted in LTLF.

The above-discussed works of literature show that two studies [20,21] address a comprehensive review on STLF, [23] addresses forecasting models based on LTLF. The study in [24] was entirely dedicated to STLF. Only a fraction (10\%) of above systematic review studies included STLF, MTLF and LTLF papers in their review; however, as argued in [10], the lead time (forecasting interval) is a factor that positively 
influences the performance of a chosen model for EED forecasting studies. Again, a high percentage of these studies $[10,12,17-22,24]$ concentrated on the methods (models), input parameter, and timeframe. Nevertheless, Wang et al. [19] revealed that the primary factors that influence EED forecasting models are property (characteristic) parameters of the building and weather parameters include. Besides, these parameters are territorial dependant and cultural bond. Thus, the weather pattern is not the same world-wide neither do we use the same building architecture and materials globally.

Notwithstanding, a higher percentage of previous systematic review studies overlooked the origin of studies and dataset of EED forecasting paper. Also, only a few studies $[12,17,19]$ that examined the evaluation metrics used in EED forecasting models. However, as pointed out in [17], there is no single validity index that can correctly deal with any dataset and offer better performance always.

Despite all these review studies [10, 12, 17-22, 24] on electricity load forecasting, a comprehensive systematic review of electricity load forecasting that takes into account all possible factors, such as the forecasting load (commercial, residential and combined), the forecast model (conventional and AI), model evaluation metrics and forecasting type (STLF, MTLF, and LTLF) that influences EED forecast models is still an open gate for research. Hence, to fill in the gap, this study presents an extensive systematic review of state-of-the-art literature based on electrical energy demand forecasting. The current review is classified according to the forecasting load (commercial, residential, and combined), the forecast model (conventional, AI and hybrids), model evaluation metrics, and forecasting type (STLF, MTLF, and LTLF). The Preferred-Reporting Items for Systematic-Review and Meta-Analysis (PRISMA) flow diagram was adopted for this study based on its ability to advance the value and quality of the systematic review as compared with other guidelines [16, $25,26]$. The current study contributes to knowledge as follows:

1. A comprehensive and detailed assessment of previous state-of-the-art studies on electricity demand forecasting; based on used methods, timeframe, the train and test split of data, error, and accuracy metrics applied to forecast.

2. We present a concise summary of the useful characteristics of compared techniques in electric load forecasting.

3. We identified the challenges and opportunities for further studies in electric load forecasting.

The remaining sections of the current paper are structured as follows. "Methodology" section presents the methods and materials used in the current study. "Data collection" section presents the results and a detailed discussion of the outcomes, and "Study framework" section presents the summary of findings and direction for future studies. 


\section{Methodology}

The current study presents a systematic review of pertinent literature on electrical energy forecasting.

\section{Data collection}

A total of eighty-one (81) state-of-the-art research works published in journals, conferences, and magazines, and student's thesis relevant to the scope of the current study were downloaded from the internet, thus using keywords and terms which included AI, Electricity Prediction (EP), Energy Forecasting (EF), Machine Learning (ML), and combination of AI and EP, AI and EF, ML and EP, ML and EF. Each downloaded literature was then carefully studied and categorised into the two methods of electrical load forecasting data-driven (artificial intelligence) methods and engineering methods.

\section{Study framework}

According to Moher et al. [16], the quality of every systematic review is based on building protocol, which outlines the justification, hypothesis, and planned methods of the investigation. However, only a few systematic review study reports of their framework. A detailed, well-described structure for systematic reviews facilitates the understanding and evaluation of the methods adopted. Hence, the PRISMA model [26] was adopted in this study (Fig. 1). As shown in Fig. 1, the PRISMA presents the flow of information from one stage to another in a systematic review of the literature and gives the total number of the research identified, excluded, and included and the reasons for inclusion and exclusions.

The PRISMA flow diagram involved five (5) phases, as shown in Fig. 1. Phase 1 consists of outlining the review scope, developing questions, and inclusion or exclusion. Phase

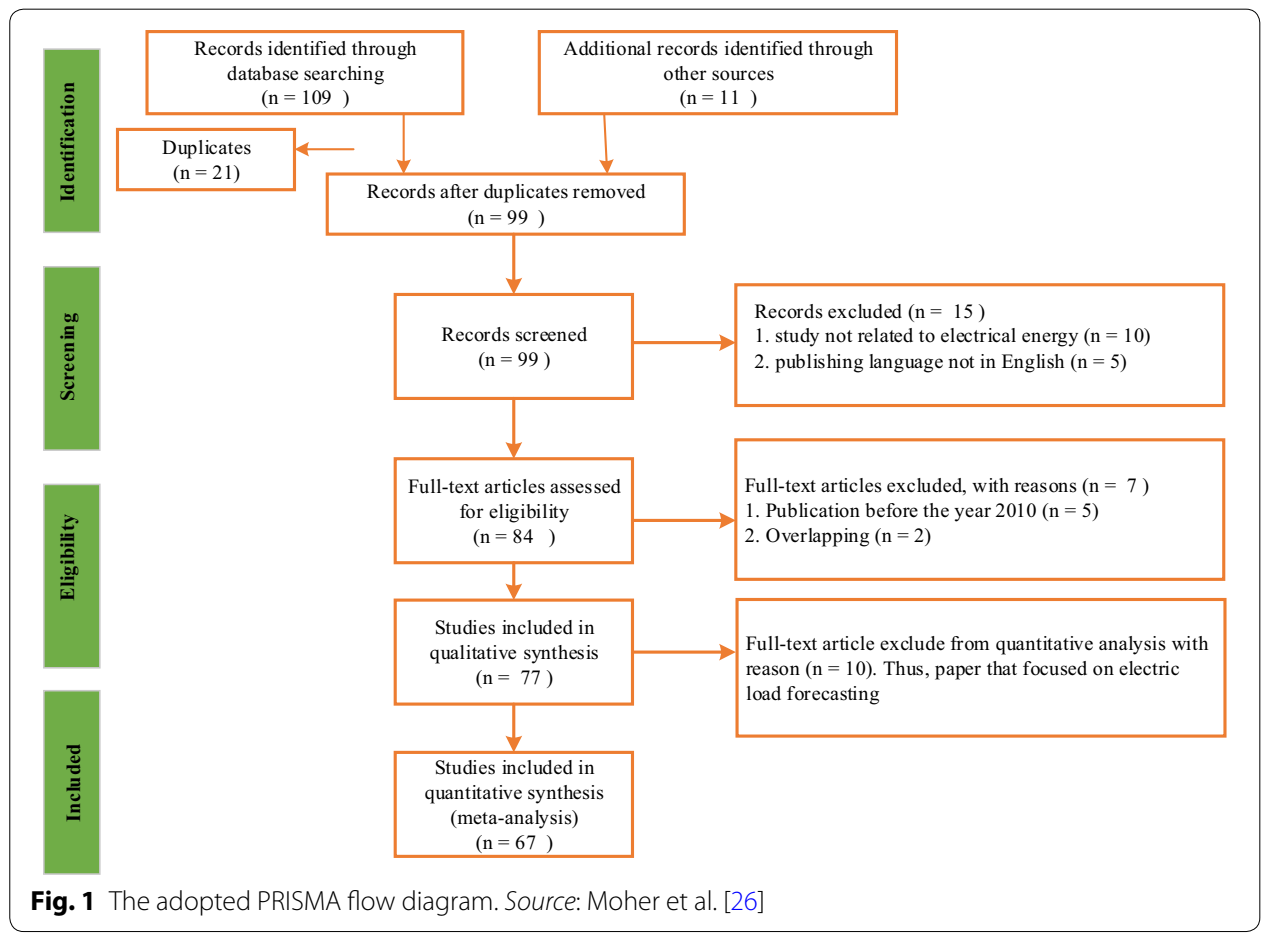


2 searches the literature with keywords to identify potential studies. Phase 3 includes determining the addition of a paper by screening its abstracts if it meets inclusion criteria. While phase 4 includes characterisation of paper for mapping by keywords. This review aimed to document an overview of research in the field of electric load forecasting to make way for future studies. As a result, a fifth (5) step offers an in-depth quantitative synthesis (meta-analysis) of studies included in the review.

Our search of literature retrieved one hundred and one (109) papers from online journals and eleven (11) from under sources, making one hundred and twelve (120) records in all (see Fig. 1). Of the 120 records, 21 were duplicates, hence, removed leaving ninetyone (99) record shortlisted for the screening stage. At the screening stage, fifteen (15) records were removed; thus, studies that were not related to electrical energy, and those that the primary publication language was not in English. Leaving eighty-four (84) records, of the 84 records, we further remove five (5) more records that were published before the year 2010, two (2) record omitted due to overlapping, this reduced eligible papers for analysis to seventy-seven (77). The 77 papers were used for the qualitative analysis. Ten (10) records that presented a review of electric load forecasting were also removed, and the remaining (67) were used for the quantitative analysis, as shown in Fig. 1.

\section{Results and discussion}

Electrical energy consumption can be classified as residential (domestic), commercial (non-residential), or industrial. Residential or domestic refers to the home or a dwelling where people globally live from day-to-day. At the same time, commercial consumers are the business and industries that require massive supply than residential users for their businesses [27]. Selected literature was on electric load forecasting was classified into two main categories AI methods and engineering methods. However, each category was further grouped into residential and commercial or combined (residential and commercial), and the outcomes are presented. A total of seventy-seven (77) papers were eligible for the qualitative analysis, while sixty-seven (67) were included in the quantitative analysis, as already discussed above in this study.

\section{Al methods used in electrical energy demand forecasting}

This section presents the studies that were based on AI techniques.

\section{Combined (commercial and residential)}

Most works on EED forecasting sought to forecast the total load (residential and commercial) demand on the supplying authority. This section presents the selected studies that fell in this category of electric load forecasting.

\section{Hybrid models}

In a way to harness the strength in different machine learning techniques, some researchers sought to hybrid two or more ML techniques to improve the forecasting accuracy of their models.

A short-term (next-day) EED forecasting model based on the historical meteorological parameter to forecast the future load on the Greek Electric Network Grid using Support 
Vector Machine (SVM), ensemble XGBoost, Random Forest (RF), k-Nearest Neighbours (KNN), Neural Networks (NN) and Decision Trees (DT) was proposed by [28]. The mean absolute percentage error (MAPE) was used as a performance metric for comparison among selected models by the author. The study achieved a reduction in prediction error or $+4.74 \%$ compared with the Operator of the Electricity Market in Greece predictions. In other studies [29], long-term (10 years) EED forecasting using NN and Autoregressive Integrated Moving Average (ARIMA) was proposed to forecast the EED of Kuwait. Weather temperature and humidity, average salary, gross domestic, oil price, population, residence, passengers, currency earning rate, and economic factors like (total import and export in USD) were used as independent variables. The study concluded that NN outperformed ARIMA and weather parameters were found to be more significant than average salary, gross domestic and oil price.

A Particle Swarm Optimisation (PSO) and Differential Evolution (DE) for forecasting the Andhra Pradesh Grid using weather parameters were presented [6]. The reported concluded that better prediction accuracy was achieved with PSO and DE than the conventional time series forecast model. In another study, a Curve Fitting Algorithm (CFA) was proposed for forecasting electricity power demand for an hour/day/week/month [30]. Their study shows that future electricity demand can be effectively forecasted based on past demand. In a process to increase forecasting accuracy, historical electricity data combined with Twitter data was used as an input variable to hybrid ANN and SVM forecast model to forecast the electricity consumption in Dutch [14]. The authors compared the performance of ANN to SVM and concluded that the ANN outperformed the SVM. On the other side, the SVM performance in accuracy increases in long-term forecasting. The authors again admit that inclusion of weather data as input could not increase model performance. Similarly, in Ghana, an attempt to predict the 30-day ahead EED demand of the Bono region using a hybrid ML (MLP, SVM, and DT) based on historical weather and electricity demand was made [7]. The authors achieved 95\% prediction accuracy; however, it affirms that the inclusion of household lifestyle as an input variable will improve prediction accuracy.

The hybrid of ML (SVM and RF) and time-series models Generalized Linear Model (GLM) and ARIMA forecast model was proposed for predict electricity consumption in South Africa based on the historical electricity price, load demand, and weather parameter [31]. The outcome of the study showed that the ARIMA-GLM combination performs better for long-term forecasting. Similarly, a combination of quantum search with SVM (quantum computing and the chaotic mechanics) for forecasting yearly EED in Taiwan was presented [32]. The empirical analysis revealed that the proposed model exhibits considerably enhanced forecasting performance than other SVM-based forecasting models. A hybrid of mode decomposition (EMD), PSO, and SVM model was present for forecasting short-term EED demand of the Australian electricity market [33]. Sulandari et al. [34] proposed a hybrid of ANN and Fuzzy algorithm and a recurrent formula (LRF) to predict electricity demand in Indonesian. The study results showed that the hybrid model performed well with low values of RMSE. Likewise, in [35], a hybrid of clustering technique (K-means) and ARIMA forecasting model was presented to forecast university buildings electricity demand. Paper revealed that the hybrid model outperformed the ARIMA model alone as a forecast model. 


\section{ANN models}

An artificial neural network (ANN)-based forecast model for short-term forecasting of Chhattisgarh State electricity demand was proposed [36], using historical weather data as input variables. The results conclude that ANN can efficiently forecast electricity demand. Likewise, an ANN model was applied to forecast the short-term electricity demand of the Iraqi National Grid [37]. The authors achieved high accuracy and a reasonable error margin. In a similar study, a DT algorithm forecast model was proposed for forecasting future EED on the Yola/Jimeta power transmission company using weather parameters. Again, a short to medium term EED forecasting using deep machine learning (ML) algorithm long short-term memory (LSTM)based neural network enhanced with genetic algorithm (GA) for feature selection was proposed [9], to forecast France metropolitan's electricity consumption. The mean absolute error (MAE) and root-mean-square error (RMSE) were used as the performance metrics, and the weather parameter was used in the independent variable. Their results affirm that weather parament is very useful in forecasting future electricity demand.

An enhanced Convolutional Neural Network (CNN) and enhanced SVM-based forecast model was presented for forecasting electricity price and load forecasting using [38]. Despite the enhancement made by authors, they recommended additional enhancement of classifiers to improve prediction. In a similar study, an ANN model to forecast consumer demand in North Cyprus was proposed [39]. Their results affirm the ability for ANN effective automatic modelling of electricity; however, the study concluded this could be achieved when the training and testing datasets are meaningful. A forecast model using a deep belief network using historical EED of Macedonian (2008-2014) was proposed to forecast a short-term (1 day) EED. The outcome of the forecast shows a reduction in MAPE by $8.6 \%$ by the proposed model compared with traditional techniques [2]. In Young et al. [40], an ANN with Bayesian regularisation algorithm-based model for short-term load forecasting of commercial building electricity usage was carried out. An ANN and hybrid methods to forecast electricity consumption of Turkey were proposed Aydogdu et al. [41]. The proposed model gave an average absolute prediction error of $2.25 \%$.

Khwaja et al. [11] presented an ensemble ANN predictive model to enhance shortterm electricity load forecasting. Different from existing studies, the authors combined both bagging and boosting techniques to train bagged-boosted ANNs. The study results showed that the proposed ensemble technique offered a reduction of both variance and bias compared to a bagged ANN, single ANN, and boosted ANN. Also, Ahmad et al. [42] combined Extreme Learning Machine and an enhanced Support Vector Machine to forecast short-term electricity demand. The study outcome showed that the proposed hybrid model outperformed other state-of-the-art predictive models in terms of performance and accuracy. Atef and Eltawil [43] proposed a deep-stacked LSTM forecasting model to forecast electricity demand. The paper reported that bidirectional (Bi-LSTM) networks outperformed unidirectional (Uni-LSTM) in terms of forecasting accuracy. Also, a generalised regression Neural Network (GRNN) predictive model was proposed in [44] to predict short-term 
electricity demand. The study results showed that a GRNN of 30 neurons offered better prediction accuracy in terms of MAPE and MAE than a GRNN of 10 neurons.

\section{Fuzzy logic models}

A Fuzzy Logic (FL)-based forecasting model for the next-day electricity demand in Albania was presented [45]. The time, the historical and forecasting value of the temperature and the previous day load (L) served as the independent variables for the forecast of the next-day consumption. The outcome of the study yielded accurate forecasting by the FL model. Motepe et al. [46] proposed an adaptive neuro-fuzzy inference system (ANFIS) model for forecasting South African electricity demand. The author concluded that adding temperature as an input parameter to the proposed model did not enhance forecast accuracy, as typically expected.

\section{SVM models}

A new economy (stock indices) reflecting the STLF model for electricity demand forecasting was proposed [47]. The authors attempted to forecast the future demand for electricity based on the Taiwan Stock Exchange Capitalization Weighted Stock Index (TAIEX) data. The study revealed a significant relationship between stock market function and energy demand, which was helpful to financial analysis wanting to do reverse engineering.

\section{Residential load forecasting}

A survey in 2019 shows that EED forecasting, especially short-term load forecasting for individual (residential) electricity customers, plays an increasingly essential role in the future grid planning and operation Kong et al. [51]. Similarly, the outcome of Leahy and Lyons [48] affirms that water heating styled used by a household is even more essential than the number of electrical appliances when explaining domestic electrical energy usage. Furthermore, a study in Portugal shows that residential electricity consumption since 1990 has been increasing more rapidly than the Gross Domestic Product (GDP) per capita [49]. Therefore, studies that focused on forecasting residential EED is of necessity. However, the results revealed that eight (8) out of the sixty-seven (67) representing (11.94\%) reviewed works focused on residential electricity forecasting, and a section of these studies is presented.

An hourly prediction of residential energy consumption using RF and SVM was proposed by Hedén [50], using 187 households in Austin. Error metrics are mean bias (MB), coefficient of variance $(\mathrm{CV})$, and MAPE. The study achieved better forecasting accuracy compared with traditional time series models. Again, an LTSM for short-term residential load forecasting based on residential meter reading [51] and resident behaviour learning [52] was studied. The outcomes of these works showed the effectiveness of the LTSM in electricity demand forecasting.

In the same way, linear regression, RF, and SVM predictive model (data-driven) for estimation of city-scale energy use in buildings were proposed by Kontokosta and Tull [53]. The outcome of the study revealed that adequate electricity consumption in a building could be predicted using actual data from a moderately small subset of buildings. Likewise, unsupervised ML algorithms such as Self-Organising Maps (SOM), k-means, 
and k-medoid were used to cluster residential electricity based on their trend of electricity use within the day [54]. The study found that households and how they use electricity in the home can be categorised based on specific customer characteristics. Mcloughlin et al. [55] examined residential electricity consumption patterns in Irish based on occupant socio-economic and dwelling variables. The variable examined includes the number of bedrooms, dwelling type, age of household head, social class, household composition, and water heating.

The study outcome showed a positive association between maximum demand periods and several household appliances, especially dishwashers, electric cookers tumble dryers, with electric cook topping. The study further found that the time of use of electrical appliances was dependent on occupant characteristics, and younger occupants of a household tend to use more electricity than the older. Alike, an ARIMA, NN, and exponential smoothening forecast model were proposed for forecasting household electricity demand [56]. The authors concluded that forecasting accuracy varies considerably depending on the choice of forecasting techniques/tactic and configuration/selection of input parameters. Again, a neural network model for predicting residential building energy consumption was proposed by Biswas et al. [57]. The outcome of the study showed that models based on OWO-Newton algorithms and Levenberg-Marquardt outperformed previous literature.

\section{Engineering and traditional electrical energy demand forecasting}

This section presents the electrical energy demand forecasting study based on the traditional time-series and engineering models.

A predictive model for the prediction of medium-term (1-year) electricity consumption of general households based on the lifestyle of the household using Lasso and Group Lasso was proposed [58]. Their results showed that household lifestyle such as family composition, age, and house-type is good predictors of electricity consumption in a home. Similarly, in Nigeria, an attempt was made to estimate the electricity demand of residential users to support energy transitions using the engineering approach, such as calculating the total power consumed in a household based on the power rating of appliance and their duration of use [59]. The study concludes that the proposed system can serve policymaking in Nigeria to improve the financial sustainability of the energy supply structure. An extended Autoregressive Distributed Lag (ARDL) model was proposed to estimate residential electricity consumption per capita demand function, which depends on the GDP per capita in Algeria [60]. The study concluded that promoting financial growth among citizens of Algeria would reduce electricity consumption, since wealthier people (higher income earners) mighty use of more efficient appliances.

In Bogomolov et al. [61], authors used general public dynamics derived data from cellular network and energy consumption dataset to predict the next-day energy demand. The study could serve a model to enhance the energy meter to promote energy conservation. Likewise, the historical data of on-off times of residential appliances were used to predict the next-day electricity demand using Bayesian inference [62]. The study concluded that historical electricity consumption data only is not adequate for a decent eminence hourly forecast. 
Also, an ARIMA and Holt-Winter model was proposed for forecasting the national electricity consumption of Pakistan from 1980 to 2011 [3]. The study revealed that the demand in household energy consumption would higher as compare with all other sectors. Jain et al. [63] proposed an ARIMA forecaster for forecasting electricity consumption. The proposed model achieved a MAPE of $6.63 \%$. The authors concluded that the ARIMA model has the potential of computing in EED forecasting with other forecast techniques. Integration of three (3) forecasting model, long-range energy alternative planning (LEAP), ARIMA, and Holt-Winter, was proposed for forecasting long-term energy demand in Pakistan [64]. The study would be valid for energy supplies for accurate estimation of users' demand in the future. The combination of Extreme Learning Machine and Multiple Regression for forecasting China's electricity demand was proposed [65]. A quantile regression (QR) model for long-term electricity demand forecasting in South Africa within 2012 and 2015 was presented by Mokilane et al. [66]. The model was helpful to power distribution industries in the country.

An attempt was made to forecast the electricity consumption of Ghana by 2030 using ARIMA-based model. The study outcome projected that Ghana's electricity consumption would grow from 8.5210 billion kWh in 2012 to 9.5597 billion kWh in 2030 [67]. However, in 2017, a report by the energy commission revealed that electricity consumption was 14,247 GWh [68]. A generalised additive model was adopted for forecasting medium-term electric energy demand in a South African power supply system [69]. The outcome of the study revealed a useful application of the proposed model in the power generation and distribution industries in the country. An investigation between the association of causal nexus and (environmental pollution, energy use, GDP per capita, and urbanisation) in an attempt to forecast Nigeria's energy use by 2030 was carried out using the ARIMA and ETS models [70]. The study outcome showed better forecasting accuracy by both models, and a high rise in energy demand was observed.

Multiple regression analysis approaches for forecasting the yearly electricity demand in commercial sectors and electricity access rates in rural and urban households in some selected West African countries, which included Ghana, were carried out by Adeoye and Spataru [71]. The study showed that there is a very high variation in hourly electricity demand in the dry seasons. Their results affirm Nti et al. [7] report that the demand pattern of electricity in Ghana is highly dependent on the month of the year. In a way, one can say there is a partial agreement in these two studies. A time-series regression model for forecasting South African's peak load demand was presented [72]. Experimental fallouts indicated that when the temperature is included as an input parameter, improvement in accuracy by the forecast model was realised. ARIMA-based predictive model for predicting both sectoral and total electrical energy consumption of Turkey for the next 15 years was proposed [73]. The study points out that the demand for electrical energy in agriculture sectors, transport, public service, residential, and commercial will keep increasing.

Similarly, partially linear additive quantile regression models for forecasting shortterm electricity demand during the peak-demand periods (i.e., from 6:00 to 8:00 pm) were carried out in South African [74]. The authors found out that the use of the 
proposed system in power utility industries for the planning, scheduling, and dispatching of electricity activities will result in a minimal cost principally during the peak-period hours. Caro et al. [75] predicted the Spanish electricity demand using the ARIMA model. The study achieved an improvement in the short-term predictions of electricity demand with less computational time. A short-term electricity load forecasting model based on dynamic mode decomposition was proposed in [76], the proposed model showed better stability and accuracy compared with other predictive models.

\section{Quantitative analysis of findings}

The descriptive statistics of the study outcome is presented under this section with tables and charts.

\section{Algorithms used for forecast models}

As part of the aim of the review, we sought to find out the most used algorithms in electricity forecasting models. The most top nine (9) used algorithms found in the sixty-seven (67) article are presented (Fig. 2); this includes only algorithms that were used in more than a single paper. The study outcome revealed that $90 \%$ out of the top nine algorithms were AI-based, with ANN representing 28\% of AI models used in electricity forecasting. Besides, the ANN models were primarily used for STLF where electrical energy consumption patterns are more intricate than LTLF. The traditional AFRIMA recorded $17.5 \%$ due to its efficiency in LTLF, where load fluctuations and periodicity are less critical.

Additionally, a high percentage of regression models is used for LTLF prediction. The study outcome shows how AI is applied in various sectors of the economy to improve efficiency and profitability. Also, we observed that the SVM, PSO and

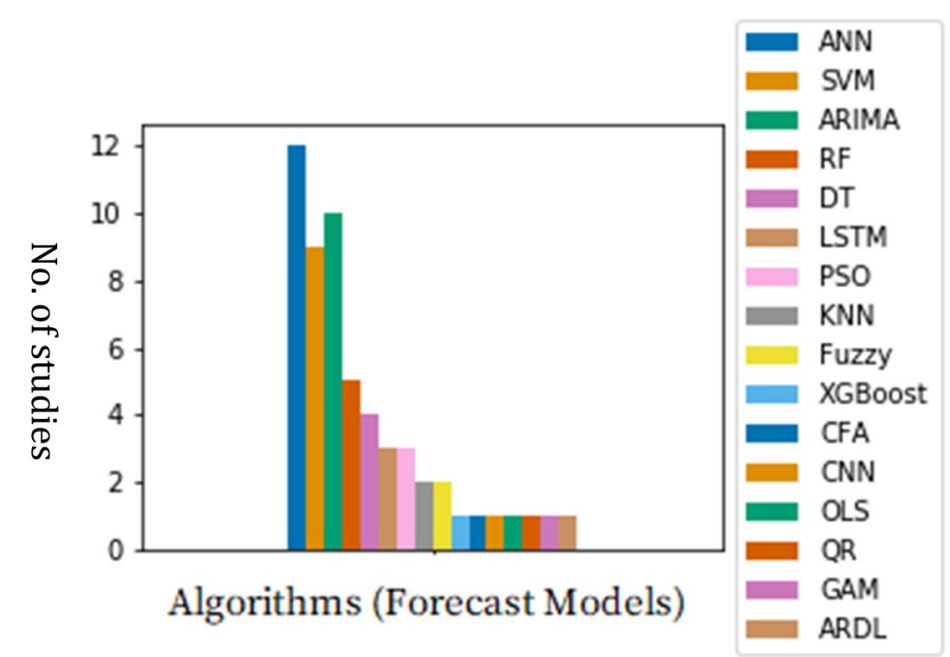

Fig. 2 Top nine (9) most used algorithms for electricity forecasting 
Table 1 Studies and their origin (for $N=67$ papers)

\begin{tabular}{lll}
\hline Continent & No. of studies & Percentage (\%) \\
\hline Europe & 21 & 31.34 \\
Africa & 12 & 17.91 \\
Asia & 13 & 19.40 \\
Not stated & 13 & 19.40 \\
Australia (or Oceania) & 5 & 7.46 \\
North America & 3 & 4.48 \\
\hline
\end{tabular}

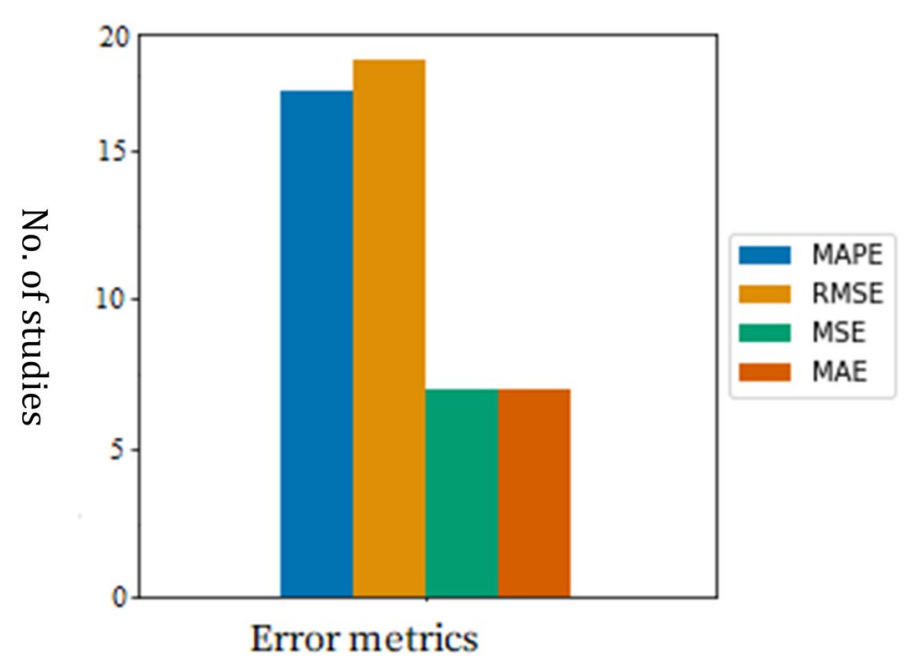

Fig. 3 Most used error metrics in electricity load forecasting

Fuzzy are gaining more popularity in recent studies, a sign of increasing attention of researchers on these algorithms for EED forecasting.

\section{Study origin}

Table 1 shows the studies and their origin (countries). The origin of surveyed studies was examined, in order to find the linkage between the power crisis in the continent and studies on electrical energy demand forecasting. Concerning geographical coverage, it was found that a high number of studies (31.34\%) were carried out in Europe, 17.91\% in Africa and 19.40\% in Asia. Making Africa the third-highest, however, interestingly, most of the studies in Africa were carried in South Africa (five representing 41.67\%), three (3) representing $25 \%$ in Ghana, with the rest from Nigeria and other African countries. The energy crisis that hit Europe in 2008, reported by [77], can be attributed to the numerous studies in electricity forecast, as shown in Table 1. It was further observed that 2 out of 3 studies in Ghana were based on national or regional electricity demand forecasting (one long-term, one short-term (1 month)). While the third study aimed at identifying the relationship between electricity demand and economic growth. Thus, it suggests that the limited number of studies in electricity 
demand forecasting by both academicians and professionals in this field might have partially contributed to the power shortages facing the nation, which in 2015 resulted in "Domsur".

\section{Used evaluation metrics}

The performance of every forecasting model is examined based on the difference in error between the actual value $(y)$ and the predicted value $(\hat{y})$. Several of these metrics were identified in the literature. However, we examined the most used in electrical energy demand forecasting to enable new beginners in the field of electricity demand forecasting to have a firm grip on which to apply in their study. Figure 3 shows the top four (4) most used error metrics in two or more studies. The results revealed that rootmean-square error RMSE (38\%) was the most used error metric among EED forecasters, followed by mean absolute percentage error MAPE (35\%). Due to the effectiveness in measure predictive model performance and their usefulness for short-term prediction, it was also observed that the MAPE is a standard primary metric as it is easy to both calculate and understand. The results affirm the findings in $[12,19,22]$ that MAE, MAPE, and RMSE are the most commonly used evaluation metrics in EED forecasting model.

\section{Forecast type}

Based on short-term load forecasting (STLF), medium-term load forecasting (MTLF), and long-term load forecasting (LTLF), it was observed that $80 \%, 15 \%$, and $5 \%$ of the electrical energy demand forecasting were STLF, MTLF, and LTLF, respectively. The massive number of studies is based on STLF as compared with LTLF and MTLF call for further studies into the challenges associated with LTLF and MTLF electricity load forecasting. Again, $80 \%$ STLF forecasting affirms the 38\% use of RMSE error metric, since it is for STLF forecasting. The results affirm the report in [22] that $43.6 \%$ of electricity forecasting are short-term prediction.

\section{Model input parameters}

The efficiency of every predictive model is believed to be partially dependent on the independent (input) variable [7]. At this level, the focus was to examine the different input variables used for electricity load forecasting. The type of independent variables (input features) used by electricity load forecasters was also examined. The current study observed that sixty (60) out of the sixty-seven (67) papers made known the input parameter to the proposed model. Table 2 presents the type of input variables and the percentage of studies that utilised it. It was observed that a high percentage $(50 \%)$ of electricity demand forecasting was based on weather parameters; next was the historical electricity

Table 2 Parameter used in electricity load forecasting

\begin{tabular}{lcl}
\hline Input variables & No. of studies & Percentage (\%) \\
\hline Weather and economic & 30 & 50 \\
Household lifestyle & 5 & 8.33 \\
Historical energy consumption & 23 & 38.33 \\
Stock indices & 2 & 3.33 \\
\hline
\end{tabular}


consumption pattern. The outcome exposed that little attention is given to household lifestyle in electricity demand forecasting. However, Nishida et al. [58] argue that residential (domestic) energy consumption differs depending on the lifestyle of the family. Family lifestyle, according to [56], cannot be undermined in electrical load forecasting. According to these studies, these factors include the life stage family composition, house type, age, home appliances possessed and their usage, family income, cultural background, social life, and lifestyle habits, which include how long to stay at home and how to spend holidays. The observations open the opportunity for further studies on the association between EED and household lifestyle.

\section{Conclusions}

The current study sought to reviewed state-of-the-art literature on electricity load forecasting to identify the challenges and opportunities for future studies. The outcome of the study revealed that electricity load forecasting is seen to be complicated for both engineers and academician and is still an ongoing area of research. The key findings are summarised as follows.

1. Several studies (90\%) have applied AI in electrical energy demand forecasting as compared with traditional engineering and statistical method (10\%) to address energy prediction problems; however, there are not enough studies benchmarking the performance of these methods.

2. There are few studies on EED forecasting in Africa countries (12 out of 67). Though the continent has progressive achievement in the creation of Regional Power Pools (PPP) over the last two decades, the continent still suffers from a lousy power network in most of its countries, leaving millions of people in Africa without electricity.

3. Temperature and rainfall as an input parameter to the EED forecasting model are seen to have a divergent view. At the same time, some sections of research recorded an improvement in accuracy and reported no improvement in accuracy when introduced and input. However, the current study attributes this to the difference in automorphic temperature globally and the different economic status among countries. An additional investigation will bring more clarity to the literature.

4. This study revealed that EED forecasting in the residential sector had seen little attention. On the other hand, Guo et al. [78] argue that the basic unit of electricity consumption is home.

5. It was observed that there had been a global increase in residential electricity demand, this according to the report in [49] can be attributed to the growing rate of buying electrical equipment and appliances of low quality due to higher living standards. However, a further probe into Soares et al. [49] assertion will bring clarity to literature because of the discrepancy in opinions in literature.

6. Lastly, the study revealed that there is a limited number of studies on load forecasting studies in Ghana. We, therefore, recommend rigorous researchers in this field in the country to enhance the economic growth of the country. 
Our future study will focus on identifying the relationship between household lifestyle factors and electricity consumption in Ghana and predict load consumption based on identified factors since it is an area that has seen little or no attention in Ghana.

\begin{abstract}
Abbreviations
EED: Electrical energy demand; Al: Artificial intelligence; ANN: Artificial neural network; RMSE: Root-mean-square error; MAPE: Mean absolute percentage error; VSTLF: Very short-term load forecasting; STLF: Short-term load forecasting; MTLF: Medium-term load forecasting; LTLF: Long-term load forecasting; PRISMA: Preferred-reporting items for systematicreview and meta-analysis; EP: Electricity prediction; EF: Energy forecasting; ML: Machine learning; SVM: Support vector machine; RF: Random forest; KNN: k-nearest neighbours; NN: Neural networks; DT: Decision trees; ARIMA: Autoregressive integrated moving average; PSO: Particle swarm optimisation; DE: Differential evolution; CFA: Curve fitting algorithm; GLM: Generalised linear model; LSTM: Long short-term memory; GA: Genetic algorithm; MAE: Mean absolute error; CNN: Convolutional neural network; FL: Fuzzy logic; ANFIS: Adaptive neuro-fuzzy inference system; GRNN: Generalised regression neural network; TAIEX: Taiwan stock exchange capitalisation-weighted stock index; GDP: Gross domestic product; MB: Mean bias; CV: Coefficient of variance; SOM: Self-organising maps; RDL: Autoregressive distributed lag; LEAP: Longrange energy alternative planning; QR: Quantile regression.
\end{abstract}

\title{
Acknowledgements
}

Not applicable.

\section{Authors' contributions}

We, at this moment, declare that IKN and MT contributed to the design and implementation of the research. MT, IKN ON-B and AFA contributed to the analysis of the results and the writing of the manuscript. The manuscript has been read and approved by all named authors, and there are no other persons who satisfied the criteria for authorship but are not listed. All authors read and approved the final manuscript.

Funding

The authors declare that they did not receive any funding for this research.

Availability of data and materials

All data generated or analysed during this study are included in this published article.

\section{Competing interests}

The authors of the current study declare that they have no competing interests.

\section{Author details}

${ }^{1}$ Department of Computer Science, Sunyani Technical University, Box 206, Sunyani, Ghana. ${ }^{2}$ Department of Electrical and Electronic Engineering, Sunyani Technical University, Sunyani, Ghana. ${ }^{3}$ Department of Computer Science and Informatics, University of Energy and Natural Resources, Sunyani, Ghana.

Received: 6 April 2020 Accepted: 30 August 2020

Published online: 09 September 2020

\section{References}

1. Aung SS (2015) Electric power is the main driving force for industrialization. http://www.globalnewlightofmyanmar. com/electric-power-is-the-main-driving-force-for-industrialization/. Accessed 2 Apr 2018

2. Dedinec A, Filiposka S, Dedinec A, Kocarev L (2016) Deep belief network based electricity load forecasting: an analysis of Macedonian case. Energy 115:1688-1700. https://doi.org/10.1016/j.energy.2016.07.090

3. Hussain A, Rahman M, Memon JA (2016) Forecasting electricity consumption in Pakistan: the way forward. Energy Policy 90:73-80. https://doi.org/10.1016/j.enpol.2015.11.028

4. Jevgenijs S, Joeri deW, Kochnakyan A, Vivien F (2017) Forecasting electricity demand: an aid for practitioners. http:// www.worldbank.org/energy/livewire. Accessed 15 Jun 2019

5. Zaman MU, Islam A, Sultana N (2018) Short term load forecasting based on internet of things (loT). BRAC University, Dhaka

6. Kumar CHJ, Veerakumari M (2012) Load forecasting of Andhra Pradesh grid using PSO, DE algorithms. Int J Adv Res Comput Eng Technol 1:179-184

7. Nti IK, Asafo-Adjei S, Agyemang M (2019) Predicting monthly electricity demand using soft-computing technique. Int Res J Eng Technol 06:1967-1973

8. Eeeguide.com (2014) Forecasting methodology. http://www.eeeguide.com/forecasting-methodology/. Accessed 4 Jan 2019

9. Bouktif S, Fiaz A, Ouni A, Serhani MA (2018) Optimal deep learning LSTM model for electric load forecasting using feature selection and genetic algorithm: comparison with machine learning approaches t. Energies (MDPI) 11:1-20. https://doi.org/10.3390/en11071636

10. Hammad MA, Jereb B, Rosi B, Dragan D (2020) Methods and models for electric load forecasting: a comprehensive review. Logist Sustain Transp 11:51-76. https://doi.org/10.2478/jlst-2020-0004

11. Khwaja AS, Anpalagan A, Naeem M, Venkatesh B (2020) Joint bagged-boosted artificial neural networks: using ensemble machine learning to improve short-term electricity load forecasting. Electr Power Syst Res 179:106080. https://doi.org/10.1016/j.epsr.2019.106080 
12. Hong T, Fan S (2016) Probabilistic electric load forecasting: a tutorial review. Int J Forecast 32:914-938. https://doi. org/10.1016/j.jijforecast.2015.11.011

13. Selvaraj KR, Sundararaj S, Ravi T (2013) Artificial neutral network based load forecasting and economic dispatch with particle swarm optimization. Int J Sci Eng Res 4:139-145

14. Stavast P (2014) Prediction of energy consumption using historical data and twitter. The University of Groningen, Groningen

15. Ouedraogo NS (2017) Modeling sustainable long-term electricity supply-demand in Africa. Appl Energy 190:10471067. https://doi.org/10.1016/j.apenergy.2016.12.162

16. Moher D, Shamseer L, Clarke M, Ghersi D, Liberati A, Petticrew M, Shekelle P, Stewart LA, Group P-P (2015) Preferred reporting items for systematic review and and explanation meta-analysis protocols (PRISMA-P) 2015: elaboration. Syst Rev 4:1-25

17. Zhou K, Yang S, Shen C (2013) A review of electric load classification in smart grid environment. Renew Sustain Energy Rev 24:103-110. https://doi.org/10.1016/j.rser.2013.03.023

18. Gonzalez-Briones A, Hernandez G, Corchado JM, Omatu S, Mohamad MS (2019) Machine learning models for electricity consumption forecasting: a review. In: 2019 2nd international conference on computer applications and information security, IEEE, pp 1-6. https://doi.org/10.1109/cais.2019.8769508

19. Wang Z, Li J, Zhu S, Zhao J, Deng S, Zhong S, Yin H, Li H, Qi Y, Gan Z (2019) A review of load forecasting of the distributed energy system. IOP Conf Ser Earth Environ Sci 237:042019. https://doi.org/10.1088/1755-1315/237/4/042019

20. Mele E (2019) A review of machine learning algorithms used for load forecasting at microgrid level. In: Sinteza 2019-International scientific conference on information technology and data related research, Singidunum University, pp 452-458. https://doi.org/10.15308/sinteza-2019-452-458

21. Shah RB (2019) A technological literature review on load forecasting in power system using artificial intelligence. Paripex-Indian J Res 8:14-15

22. Kuster C, Rezgui Y, Mourshed M (2017) Electrical load forecasting models: a critical systematic review. Sustain Cities Soc 35:257-270. https://doi.org/10.1016/j.scs.2017.08.009

23. Panda SK, Mohanty SN, Jagadev AK (2017) Long term electrical load forecasting: an empirical study across techniques and domains. Indian J Sci Technol 10:1-16

24. Jacob M, Neves C, Vukadinović-Greetham D (2020) Short term load forecasting. Forecast Assess Risk Individ Electr Peaks Math Planet Earth. https://doi.org/10.1007/978-3-030-28669-9_2

25. Appiahene P, Ussiph N, Missah YM (2018) Information technology impact on productivity. In: International conference on applied science and technology, Kumasi Technical University, Kumasi, Ghana, pp 122-133

26. Moher D, Liberati A, Tetzlaff J, Altman DG (2009) PRISMA Group, preferred reporting items for systematic reviews and meta-analyses: the PRISMA statement. Ann Intern Med 151:264-269. https://doi.org/10.7326/0003-4819-151-4-20090 8180-00135

27. Electricchoice.com (2016) Residential electricity for houses, apartments, condos, trailers, etc. https://www.electricchoice. com/residential-electricity. Accessed 10 Aug 2019

28. Kouroupetroglou P-N (2017) Machine learning techniques for short-term electric load forecasting. Aristotle University of Thessaloniki, Thessaloniki

29. Zakarya S, Abbas H, Belal M (2017) Long-term deep learning load forecasting based on social and economic factors in the Kuwait region. J Theor Appl InfTechnol 95:1524-1535

30. Soni A, Sharma AK (2013) Electricity load forecast for power system planning. Int Ref J Eng Sci 2:52-57

31. Angamuthu CR, Mukherjee A, Campion M, Salehfar H, Hansen T, Lin J, Ranganathan P (2018) A multi-stage price forecasting model for day-ahead electricity markets. Forecasting 1:3. https://doi.org/10.3390/forecast1010003

32. Lee CW, Lin BY (2016) Application of hybrid quantum tabu search with support vector regression (SVR) for load forecasting. Energies 9:1-16. https://doi.org/10.3390/en9110873

33. Hong WC, Fan GF (2019) Hybrid empirical mode decomposition with support vector regression model for short term load forecasting. Energies 12:1-16. https://doi.org/10.3390/en12061093

34. Sulandari W, Subanar MH, Lee PC (2020) Rodrigues, Indonesian electricity load forecasting using singular spectrum analysis, fuzzy systems and neural networks. Energy 190:116408. https://doi.org/10.1016/j.energy.2019.116408

35. Nepal B, Yamaha M, Yokoe A, Yamaji T (2020) Electricity load forecasting using clustering and ARIMA model for energy management in buildings. Jpn Archit Rev 3:62-76. https://doi.org/10.1002/2475-8876.12135

36. Ghore S, Goswami A (2015) Short term load forecasting of Chhattisgarh grid using artificial neural network. Int J Eng Dev Res 3:391-397

37. Kuhba H, Al-tamemi HAH (2016) Power system short-term load forecasting using artificial neural networks. Int J Eng Dev Res 4:78-87

38. Zahid M, Ahmed F, Javaid N, Abbasi R, Zainab KH, Javaid A, Bilal M, Akbar M, llahi M (2019) Electricity price and load forecasting using enhanced convolutional neural network and enhanced support vector regression in smart grids. Electronics 8:122. https://doi.org/10.3390/electronics8020122

39. Nwulu Nl, Agboola OP (2012) Modelling and predicting electricity consumption using artificial neural networks. In: 2012 11th international conference on environmental and electrical engineering, pp 1059-1063

40. Young TC, Raya H, Youngdeok H, Young ML, (2016) Artificial neural network model for forecasting sub-hourly electricity usage in commercial buildings. Energy Buildings 111:184-194. https://doi.org/10.1016/j.enbuild.2015.11.045

41. Aydodu G, Yildiz O (2017) Forecasting the annual electricity consumption of Turkey using a hybrid model. In: 2017 25th signal processing and communication application conference, Antalya, Turkey, pp 1-4. https://doi.org/10.1109/ SIU.2017.7960283

42. Ahmad W, Ayub N, Ali T, Irfan M, Awais M, Shiraz M, Glowacz A (2020) Towards short term electricity load forecasting using improved support vector machine and extreme learning machine. Energies 13:1-17. https://doi.org/10.3390/ en13112907

43. Atef S, Eltawil AB (2020) Assessment of stacked unidirectional and bidirectional long short-term memory networks for electricity load forecasting. Electr Power Syst Res 187:106489. https://doi.org/10.1016/j.epsr.2020.106489

44. Azar AT, Khamis A, Kamal NA, Galli B (2020) Short term electricity load forecasting through machine learning. In: Hassanien A, Azar A, Gaber T, Oliva D, Tolba F (eds) International conference on artificial intelligence computer vision 
(AICV2020). AlCV 2020. Advances in intelligent systems and computing, vol 1153, Springer, Cham. https://link.springer. com/chapter/10.1007/978-3-030-44289-7_40. Accessed 5 July 2020

45. Konica JA, Hanelli L (2016) Forecasting next-day the electricity demand based on fuzzy logic method case for. J Multidiscip Eng SciTechnol 3:6172-6180

46. Motepe S, Hassan AN, Stopforth R (2019) South African distribution networks load forecasting using ANFIS. In: Proceedings of 2018 IEEE international conference power electronic drives energy system PEDES 2018, IEEE, Chennai, India, pp 1-6. https://doi.org/10.1109/pedes.2018.8707876

47. Lin CT, Der Chou L (2013) A novel economy reflecting short-term load forecasting approach. Energy Convers Manag 65:331-342. https://doi.org/10.1016/j.enconman.2012.08.001

48. Leahy E, Lyons S (2010) Energy use and appliance ownership in Ireland. Energy Policy 38:4265-4279. https://doi. org/10.1016/j.enpol.2010.03.056

49. Soares A, Gomes Á, Antunes CH (2014) Categorisation of residential electricity consumption as a basis for the assessment of the impacts of demand response actions. Renew Sustain Energy Rev 30:490-503. https://doi.org/10.1016/j. rser.2013.10.019

50. Hedén W (2016) Predicting hourly residential energy consumption using random forest and support vector regression an analysis of the impact of household clustering on the performance accuracy. KTH Royal Institute of Technology, Stockholm

51. Kong W, Dong ZY, Jia Y, Hill DJ, XU Y, Zhang Y (2019) Short-term residential load forecasting based on LSTM recurrent neural network. IEEE Trans Smart Grid 10:841-851. https://doi.org/10.1109/TSG.2017.2753802

52. Kong W, Dong ZY, Hill DJ, Luo F, Xu Y (2018) Short-term residential load forecasting based on resident behaviour learning. IEEE Trans Power Syst 33:2016-2017. https://doi.org/10.1109/TPWRS.2017.2688178

53. Kontokosta CE, Tull C (2017) A data-driven predictive model of city-scale energy use in buildings. Appl Energy 197:303317. https://doi.org/10.1016/j.apenergy.2017.04.005

54. McLoughlin F, Duffy A, Conlon M (2015) A clustering approach to domestic electricity load profile characterisation using smart metering data. Appl Energy 141:190-199. https://doi.org/10.1016/j.apenergy.2014.12.039

55. Mcloughlin F, Duffy A, Conlon M (2012) Characterising domestic electricity consumption patterns by dwelling and occupant socio-economic variables: an Irish case study. Energy Build 48:240-248. https://doi.org/10.1016/j.enbui Id.2012.01.037

56. Veit A, Goebel C, Tidke R, Doblander C, Jacobsen H (2014) Household electricity demand forecasting-benchmarking state-of-the-art methods. arXiv:1603.00751, pp 1-10

57. Biswas MAR, Robinson MD, Fumo N (2016) Prediction of residential building energy consumption: a neural network approach. Energy 117:84-92. https://doi.org/10.1016/j.energy.2016.10.066

58. Nishida K, Takeda A, Iwata S, Kiho M, Nakayama I (2017) Household energy consumption prediction by feature selection of lifestyle data. In: International conference on smart grid communication, Dresden, Germany, pp 235-240

59. Olaniyan K, Mclellan BC (2018) Estimating residential electricity consumption in Nigeria to support energy transitions. Sustain 10:1-22. https://doi.org/10.3390/su10051440

60. Bouznit M, Pablo-Romero MP (2018) Residential electricity consumption and economic growth in Algeria. Energies (MDPI). https://doi.org/10.3390/en11071656

61. Bogomolov A, Lepri B, Larcher R, Antonelli F, Pianesi F, Pentland A (2016) Energy consumption prediction using people dynamics derived from cellular network data. EPJ Data Sci. https://doi.org/10.1140/epjds/s13688-016-0075-3

62. Huber P, Gerber M, Rumsch A, Paice A (2018) Prediction of domestic appliances usage based on electrical consumption. Energy Inform 1:265-428

63. Jain PK, Quamer W, Pamula R (2018) Electricity consumption forecasting using time series analysis. In: Singh M, Gupta P, Tyagi V, Flusser J, Ören T (eds), Advanced computer data science ICACDS 2018. Communications in computer and information science, Springer, Singapore, Singapore. https://doi.org/10.1007/978-981-13-1813-9_33

64. Rehman USA, Cai Y, Fazal R, Das Walasai G, Mirjat NH (2017) An integrated modeling approach for forecasting long-term energy demand in Pakistan. Energies 10:1-23. https://doi.org/10.3390/en10111868

65. Liang Y, Niu D, Cao Y, Hong WC (2016) Analysis and modeling for China's electricity demand forecasting using a hybrid method based on multiple regression and extreme learning machine: a view from carbon emission. Energies 9:1-22. https://doi.org/10.3390/en9110941

66. Mokilane P, Galpin J, Yadavalli VSS, Debba P, Koen R, Sibiya S (2018) Density forecasting for long-term electricity demand in South Africa using quantile regression. S Afr J Econ Manag Sci 21:1-14. https://doi.org/10.4102/sajems.v21i1.1757

67. Sarkodie SA (2017) Estimating Ghana's electricity consumption by 2030: an ARIMA forecast, energy sources. Part B Econ Plan Policy 12:936-944. https://doi.org/10.1080/15567249.2017.1327993

68. Energy Commission, Energy Commission, Ghana 2018 Energy (Supply and Demand) Outlook for Ghana, Accra, 2018. http://www.energycom.gov.gh/planning/data-center/energy-outlook-for-ghana. Accessed 1 Feb 2020

69. Sigauke C (2017) Forecasting medium-term electricity demand in a South African electric power supply system. J Energy S Afr. https://doi.org/10.17159/2413-3051/2017/v28i4a2428

70. Asumadu-Sarkodie S, Owusu PA (2016) Forecasting Nigeria's energy use by 2030, an econometric approach, Energy Sources. Part B Econ Plan Policy 11:990-997. https://doi.org/10.1080/15567249.2016.1217287

71. Adeoye O, Spataru C (2019) Modelling and forecasting hourly electricity demand in West African countries. Appl Energy 242:311-333. https://doi.org/10.1016/j.apenergy.2019.03.057

72. Sigauke C, Chikobvu D (2016) Peak electricity demand forecasting using time series regression models: an application to South African data. J Stat Manag Syst 19:567-586. https://doi.org/10.1080/09720510.2015.1086146

73. Ozturk S, Ozturk F (2018) Prediction of energy consumption of Turkey on sectoral bases by Arima model. Energy Econ Lett 5:23-30. https://doi.org/10.18488/journal.82.2018.51.23.30

74. Lebotsa ME, Sigauke C, Bere A, Fildes R, Boylan JE (2018) Short term electricity demand forecasting using partially linear additive quantile regression with an application to the unit commitment problem. Appl Energy 222:104-118. https:// doi.org/10.1016/j.apenergy.2018.03.155

75. Caro E, Juan J, Cara J (2020) Periodically correlated models for short-term electricity load forecasting. Appl Math Comput 364:124642. https://doi.org/10.1016/j.amc.2019.124642 
76. Kong X, Li C, Wang C, Zhang Y, Zhang J (2020) Short-term electrical load forecasting based on error correction using dynamic mode decomposition. Appl Energy 261:114368. https://doi.org/10.1016/j.apenergy.2019.114368

77. Auverlot D, Beeker É, Hossie G, Oriol L, Rigard-Cerison A (2014) The crisis of the european electricity system: diagnosis and possible ways forward. https://www.strategie.gouv.fr/sites/strategie.gouv.fr/files/archives/CGSP_Report_Europ ean_Electricity_System_030220141.pdf. Accessed 18 Apr 2019

78. Guo Z, Zhou K, Zhang C, Lu X, Chen W, Yang S (2018) Residential electricity consumption behavior: influencing factors, related theories and intervention strategies. Renew Sustain Energy Rev 81:399-412. https://doi.org/10.1016/j. rser.2017.07.046

\section{Publisher's Note}

Springer Nature remains neutral with regard to jurisdictional claims in published maps and institutional affiliations.

Submit your manuscript to a SpringerOpen ${ }^{\circ}$ journal and benefit from:

- Convenient online submission

- Rigorous peer review

- Open access: articles freely available online

- High visibility within the field

- Retaining the copyright to your article

Submit your next manuscript at $\gg$ springeropen.com 